\title{
Lipopolysaccharide recognition protein, MD-2, facilitates cellular uptake of $E$. coli-derived plasmid DNA in synovium
}

\author{
Jacquelyn A. Kolka \\ Andrew P. Vreede \\ Blake J. Roessler* \\ Division of Rheumatology, \\ Department of Internal Medicine, \\ University of Michigan Medical \\ School, 3560 MSRB 2, 1150 W. \\ Medical Center Dr., Ann Arbor, MI \\ 48109-0688, USA \\ *Correspondence to: \\ Blake J. Roessler, Division of \\ Rheumatology, Department of \\ Internal Medicine, University of \\ Michigan Medical School, 3560 \\ MSRB 2, 1150 W. Medical Center \\ Dr., Ann Arbor, MI \\ 48109-0688, USA. E-mail: \\ roessler@umich.edu
}

\begin{abstract}
Background Several cell types are susceptible to transfection in vivo using naked plasmid DNA. The mechanisms involved in mediating in vivo transfection are incompletely known, but evidence suggests that receptormediated endocytosis is important for specific types of cells. In this study we tested the hypothesis that residual Escherichia coli lipopolysaccharide (LPS) forms a non-covalent complex with expression plasmid DNA, and host-cellderived soluble LPS-binding proteins bind to the DNA-LPS complexes in order to facilitate receptor-mediated endocytosis.
\end{abstract}

Methods Cells from the murine synovial lining were used as an in vivo model system and in vivo luciferase imaging was used to quantify levels of transgene expression. Using a series of gene-deleted mice, the roles of LPS recognition complex proteins, lipopolysaccharide-binding protein (LBP), CD14 and MD-2, in the process of in vivo transfection were determined.

Results Luciferase expression assays revealed that mice lacking LBP or CD14 had increased luciferase expression $(p<0.023$ and $<0.165$, respectively), while mice deleted of MD-2 had significant reductions in luciferase expression $(p<0.001)$. Gene deletion of hyaluronic acid binding protein CD44 was used as a control and had no statistically significant effect on transgene expression in vivo. In muscle tissue, where neither cell surface nor soluble MD-2 is expressed, no MD-2 dependence of plasmid transfection was identified, suggesting the role of MD-2 is tissue or cell type specific. Additionally, depleting mice of macrophages showed that luciferase expression is occurring within fibroblast-like synoviocytes.

Conclusions Our data support a physical association between LPS and E. coli-derived plasmid DNA, and that in vivo transfection of fibroblast-like synoviocytes is dependent on the soluble form of the LPS-binding protein MD-2. Copyright $\odot 2005$ John Wiley \& Sons, Ltd.

Keywords lipopolysaccharide; plasmid DNA; MD-2; gene transfer

\section{Introduction}

The observation that E. coli-derived plasmid DNA can mediate transfection of specific cell types in vivo was made initially in skeletal muscle [1-5]. Subsequent studies have shown that other cell types are amenable to in vivo transfection using E. coli-derived plasmid DNA without additional delivery components. The cell types so far identified 
include thyroid follicular cells [6], salivary ductal cells [7], follicular keratinocytes [8,9], hepatocytes [10,11], and synovial fibroblasts $[12,13]$.

Many of these studies have shown that the process of DNA uptake and transfection is dose-dependent and saturable $[1,8]$. These studies all suggest that cellular uptake of E. coli-derived plasmid DNA occurs via receptor-mediated endocytosis, although the exact receptor(s) through which the DNA enters is unknown [14]. Despite the widespread use of naked plasmid DNA as a clinical gene transfer vector the mechanisms associated with plasmid DNA uptake remain poorly understood [15].

The use of $E$. coli to derive expression plasmid DNA as well as recombinant eukaryotic proteins is standard in both the academic community and the pharmaceutical industry. One of the primary safety concerns involved in the use of $E$. coli-derived biological products is contamination with Gram-negative lipopolysaccharide (LPS). LPS is a potent biological molecule that exists in the outer membrane of Gram-negative bacteria. It is composed of a polysaccharide backbone linked to a fatty acid moiety termed lipid A. The lipid A portion is composed of a hydrophilic disaccharide bound to multiple hydrophobic fatty acid chains, and upon binding to host cell membranes, induces an endotoxic reaction in humans $[16,17]$. The recognition of LPS as a potential contaminant has prompted the US Food and Drug Administration to mandate the testing of such products intended for use in humans or animals for the presence of endotoxin with the limulus amebocyte lysate test [18].

As LPS enters body fluids (e.g. serum, saliva, synovial fluid) it first interacts with the plasma protein LPS-binding protein (LBP), which catalyzes the binding of LPS to either soluble or membrane-bound CD14 [19-22]. For the body to generate an immune response signaling the presence of bacterial LPS, CD14 in turn presents LPS to TLR-4, which is the transmembrane signaling receptor for LPS [23]. TLR-4 requires a physical association with the adapter protein MD-2 for expression, distribution, and proper signaling of the presence of LPS [24-26].

In order to better understand the mechanisms responsible for in vivo transfection with $E$. coli-derived plasmid DNA, we hypothesized that residual Gramnegative LPS forms a non-covalent complex with plasmid DNA. In turn the putative LPS-DNA complex is recognized by the cellular proteins that comprise the LPS recognition complex and components that facilitate receptor-mediated endocytosis. We and others have shown previously that the synovial membrane is susceptible to in vivo transfection following intra-articular administration of expression plasmid DNA [12,27]. To test our theory of DNA uptake we have developed an in vivo synovial expression assay using a pCF1-luciferase expression plasmid and an in vivo bioluminescent imaging system. Purified plasmid was administered by intraarticular injection into the stifle joints of both wild-type control and gene-deleted mice that were deficient in individual components of the LPS recognition complex. Involvement of specific components in the process of
E. coli-derived plasmid DNA uptake was determined by quantifying changes in luciferase expression levels in vivo between the wild-type control and gene-deleted mice. The changes detected in luciferase expression using our in vivo expression assay under experimental conditions support the inference that the cellular uptake of plasmid DNA is modulated by recognition components of LPS.

\section{Materials and methods}

\section{Plasmid DNA and endotoxin testing}

The pCF1-luciferase (pCF1-Luc) plasmid, which contains the cytomegalovirus promoter/enhancer region and firefly (Photinus pyralis) luciferase gene, was purified using an alkaline lysis procedure with the CONCERT plasmid purification system (GibcoBRL, Gaithersburg, MD, USA) according to the manufacturer's instructions [28]. The plasmid was further purified via cesium chloride ultracentrifugation. Sheared salmon sperm DNA (ss-DNA; Brinkmann Instruments Inc., Westbury, NY, USA) was also isolated using cesium chloride gradient ultracentrifugation performed contemporaneously with the plasmid in order to isolate linear DNA that is similar in size to that of the plasmid. Purified plasmid and ss-DNA were tested for presence of endotoxin by a kinetic limulus amebocyte lysate assay (LAL Kinetic-QCL; Biowhittaker, Walkersville, MD, USA) prior to injections. E. coli-derived plasmid DNA used in these experiments contained less than 20 endotoxin units (EU) per milligram of DNA. All reagents used were of high molecular grade to minimize the possibility of contamination with LPS/endotoxin. Synthetic, double-stranded oligonucleotides of 25 and 32 nt were produced by Integrated DNA Technologies, Inc. (Coralville, IA, USA).

\section{NMR}

Deuterium oxide $\left(\mathrm{D}_{2} \mathrm{O}\right.$; Sigma-Aldrich, St. Louis, MO, USA) was the solvent used for all samples analyzed. The plasmid and salmon sperm DNA were prepared to a concentration of $5 \mathrm{mg} / \mathrm{ml}$, and the oligonucleotides to $1 \mathrm{mg} / \mathrm{ml}$. The LPS (Sigma-Aldrich) was prepared to $1 \mathrm{mg} / \mathrm{ml}$ and 2-fold serially diluted to generate a standard curve. The oligonucleotides were analyzed both spiked and not spiked with $0.125 \mathrm{mg} / \mathrm{ml}$ LPS. The biochemical nuclear magnetic resonance (NMR) was performed with a Bruker Avance DRX-500 NMR spectrometer with a ${ }^{1} \mathrm{H}$ NMR frequency of $500 \mathrm{MHz}$ at the Nuclear Magnetic Resonance Core (College of Pharmacy, University of Michigan, USA).

\section{Analysis of plasmid DNA for FAMEs}

To $500 \mu \mathrm{l}(1 \mathrm{mg})$ of each sample were added $100 \mu \mathrm{l}$ of a 17:0 fatty acid internal standard in $15 \mathrm{ml}$ tapered, 
acid-cleaned Pyrex test tubes with Teflon-lined screw caps. The pellet was extracted with a $1: 2: 1.5$ ratio of methanol/chloroform/ $0.1 \mathrm{M} \mathrm{KCl}$ in $50 \%$ methanol by rigorous vortexing and subsequent centrifugation. The organic phase was re-extracted with a $2.5 \times$ volume of $0.1 \mathrm{M} \mathrm{KCl}$ in $50 \%$ methanol. The organic phase was then evaporated with $\mathrm{N}_{2}$ and transmethylated with $6 \%$ methanolic- $\mathrm{HCl}$ for $3.5 \mathrm{~h}$ at $80^{\circ} \mathrm{C}$.

The sample was then prepared by one of two methods, and each was analyzed. The subsequently formed fatty acid methyl esters (FAMEs) were suspended in $200 \mu \mathrm{l}$ benzene, filtered using a $0.45 \mu \mathrm{m}$ filter, and evaporated with $\mathrm{N}_{2}$. Alternatively, the FAMEs in the methanolic-HCl were further extracted using an equivalent volume of petroleum ether, bp $30-60{ }^{\circ} \mathrm{C}$, twice. After evaporation of the sample with an $\mathrm{N}_{2}$ stream, the sample was suspended in $200 \mu \mathrm{l}$ benzene, filtered using a $0.45 \mu \mathrm{m}$ filter and evaporated with $\mathrm{N}_{2}$. The sample was resuspended in $100 \mu \mathrm{l}$ of chloroform for analysis and injected in an aliquot of either 0.6 or $1.0 \mu$ l.

The samples were analyzed using a Shimadzu gas chromatography (GC) unit model GC-14A equipped with a fused-silica Megabore DB225 column (0.53 micron diameter) from J.W. Scientific (Folsom, CA, USA). A 21 fatty acid standard (Nu-CHEK-PREP, Inc., Elysian, MN, USA) was used to calibrate the GC elution times and to identify the fatty acids. The added 17:0 fatty acid was used to quantitate the amount of fatty acid in the samples. The flame ionization detector output of the gas chromatograph was digitized by an IBM-PC computer interface (model AN-146; Alpha Products, Darien, CT, USA). Both the recording and data evaluation software were created in BASIC or FORTRAN. The sensitivity of the assay was between 0.5 and $1.0 \mu \mathrm{g} / \mathrm{sample}$.

\section{Mice}

C57BL/6J wild-type controls, B6.129-CD44 ${ }^{\mathrm{tm} 1 \mathrm{Hbg}}$ CD44deficient, C57BL/10ScN TLR-4 deficient, and B6.129S$\mathrm{Cd} 14^{\mathrm{tm} 1 \mathrm{Frm}}$ CD14-deficient mice were purchased from The Jackson Laboratory (Bar Harbor, ME, USA). LBP-deficient mice were donated by Dr. Grace L. Su (University of Michigan), and B6MD-2 -/- mice were kindly provided by Dr. Kensuke Miyake (The University of Tokyo, Tokyo, Japan). All mice were housed in the Unit for Laboratory Animal Medicine at the University of Michigan. All animal experiments were reviewed and approved by the University of Michigan Committee for the Use and Care of Animals.

\section{Intra-articular injections, bio-imaging and quantification}

Five mice from each of the genotypes described above were used. All experimental mice were treated concurrently with an equal number of wild-type mice as control. The mice were anesthetized, and the knees and surrounding area were depilated using a commercial hair remover. The mice were then injected with $30 \mu \mathrm{g}(6 \mu \mathrm{l}$ at $5 \mu \mathrm{g} / \mu \mathrm{l})$ of purified naked pCF1-Luc plasmid intra-articularly into each stifle joint. Twenty-four hours after plasmid DNA injections anesthetized mice were imaged using the In vivo imaging System (IVIS; Xenogen, Alameda, CA, USA) in the Michigan Small Animal Imaging Resource (MSAIR), Center for Molecular Imaging, Department of Radiology. Mice were imaged for a 5-min luminescent integration time before and after intra-peritoneal injection of the luciferase substrate, a luciferin potassium salt in sterile phosphate-buffered saline (PBS, $40 \mathrm{mg} / \mathrm{ml}$ ) at a dose of $150 \mathrm{mg} / \mathrm{kg}$ body weight. Mice were imaged again $15 \mathrm{~min}$ after the luciferin injection. The photons emitted from the luminescence reaction were detected as photons/second and depicted as a pseudocolor overlay on a grayscale animal image for easy visualization.

\section{Intra-muscular injections}

Two C57BL/6J mice and two B6MD-2 -/- mice were intra-muscularly injected into each quadricep with $30 \mu \mathrm{g}$ $(6 \mu \mathrm{l}$ at $5 \mu \mathrm{g} / \mu \mathrm{l})$ purified pCF1-Luc plasmid DNA as above. Exactly as described above mice were imaged using the IVIS $24 \mathrm{~h}$ post-plasmid administration and following intra-peritoneal injection of the luciferin. Expression of the luciferase protein was quantified as photons/second, and mean levels were compared between the two groups.

\section{Etoposide treatments}

Etoposide was used to deplete synovial macrophages from the synovial membrane [29] in order to determine which of the two primary cells within the synovium, synovial macrophages or fibroblast-like synoviocyte [30,31] was undergoing transfection. Wild-type C57BL/6J mice were treated with either sterile PBS $(n=4)$ or etoposide $(n=4)$ by subcutaneous injection for 3 consecutive days followed on day 4 by no treatment and intraarticular plasmid injection. On day 5 they received another dose of either PBS or etoposide a few hours prior to synovial bioluminescent imaging. The etoposide dose was $12.5 \mathrm{mg} / \mathrm{kg}$ body weight. As above, luciferase expression was quantified as photons/second, and mean values were compared between the two groups. Knee joints of both etoposide- and PBS-treated mice were removed and made into $5 \mu \mathrm{m}$ thick paraffin sections. Following treatment to block endogenous peroxidase activity, sections were stained with either purified anti-mouse CD14 antibody (BD Pharmingen, San Diego, CA, USA) or no primary antibody, and then photographed at $80 \times$ magnification.

\section{Statistical methods}

SPSS statistical software and Microsoft Excel were used to analyze all data. In the in vivo bioluminescent assay 
plasmid expression was quantified as photons/second. Pre-luciferin baseline values were subtracted from postluciferin values for each mouse. The experimental group was then compared with the control with a t-test comparing mean photons/second for each group.

\section{Results}

\section{NMR}

To analyze the association between DNA and LPS we applied biochemical NMR to pure LPS, synthetic oligonucleotides, sheared salmon sperm DNA, and E. coliderived plasmid DNA. Synthetic oligonucleotides were used to avoid any naturally contaminating endotoxin. Similarly, the salmon sperm DNA was used as a comparison of like molecular weight to the pCF1-Luc plasmid without contaminating endotoxin (although very small amounts of endotoxin are unavoidable since it is produced with $E$. coli-derived reagents). Figure 1A shows the spectra of pure LPS at a concentration of $1.0 \mathrm{mg} / \mathrm{ml}$. The oligonucleotides were analyzed at a concentration of $1.0 \mathrm{mg} / \mathrm{ml}$ without (Figure 1B) and with (Figure 1C) a $0.125 \mathrm{mg} / \mathrm{ml}$ spike of LPS. The spectrum of the pure oligonucleotide was compared to that of the oligonucleotide spiked with LPS and pure LPS by overlaying the three spectra to identify peaks representing LPS within the DNA sample (Figure 1D). There are distinct peaks near $0.7,1.1,1.2$, and 2.0 that appear both in spectra of LPS and LPS-spiked oligonucleotides and that are not in the unspiked oligonucleotides. These peaks are representative of the protons of methyl groups at the end of fatty acid chains similar to the fatty acid chains of the lipid A portion of the LPS [32]. It is this lipid portion that is known to bind to eukaryotic receptor components to generate cellular immune responses $[33,34]$. We also compared the spectra of pCF1-Luc and salmon sperm DNA to that of serial dilutions of LPS attempting to determine the stoichiometric relationship between LPS and plasmid. However, the limitations in sample detection of the spectrometer prevented analysis of the concentrations of the LPS that exist in our plasmid DNA preparation and in the commercially prepared salmon sperm DNA (data not shown). Analysis of plasmid DNA for FAMEs was designed to provide quantitative estimates of lipid A/DNA stoichiometry. However, all of the DNA samples evaluated had levels of FAMEs that were below the limits of detection ( $0.5 \mu \mathrm{g}$ per sample, data not shown).

\section{Bioluminescent in vivo imaging}

To identify the mechanism of in vivo transfection following intra-articular injection of $E$. coli-derived plasmid DNA we quantified luciferase expression in a series of gene-deleted mice. These included knockout mice for LBP, CD14, TLR-4, and MD-2, all previously characterized as important functional signaling components of the LPS recognition complex. Figure 2 shows the results of the in vivo luciferase expression assays. Expression of the luciferase is reported as photons/second, and the pseudocolor gray scale images are representative of data obtained from imaging of an individual animal.

As shown in Figure 2A, mice lacking functional LPSbinding protein, LBP, have a significant increase in luciferase expression as compared to wild-type mice. A similar, although less significant, effect was observed in CD14-deficient mice (Figure 2B). The involvement of these two LPS-binding proteins in plasmid-mediated transfection indicates that cytoplasmic uptake of pCF1Luc is linked functionally to recognition and trafficking of LPS. This data strongly suggests a non-covalent complex exists between LPS and plasmid DNA.

Figure 2C shows that mice deficient in the LPS transmembrane signaling receptor TLR-4 produced no change in luciferase expression. This suggests that TLR-4 is not directly involved in the endocytic uptake of the hypothesized LPS-DNA complex, and confirms previous data suggesting that internalization of LPS proceeds through a cellular pathway distinct from TLR-4-mediated signal transduction $[35,36]$. As a control for trafficked and membrane-bound, as well as soluble, proteins within the synovium we used the highly functionally and structurally variable protein CD44. CD44 is widely expressed in both fibroblast-like synoviocytes and chondrocytes, and functions as a receptor for hyaluronic acid, the major component of synovial fluid [37]. Because there is no data suggesting that CD44 is involved in binding to LPS we studied CD44 knockout mice to control for non-specific interactions between LPS, plasmid DNA, and synovial

Figure 1. Biochemical ${ }^{1} \mathrm{H}-\mathrm{NMR}$ spectroscopy. All samples were dissolved in $\mathrm{D}_{2} \mathrm{O}$ and analyzed at a frequency of $500 \mathrm{MHz}$ (A) pure LPS at $1.0 \mathrm{mg} / \mathrm{ml}$; (B, C) synthetic oligonucleotides of different sequences, both at a concentration of $1.0 \mathrm{mg} / \mathrm{ml}$. The oligonucleotide in (C) is spiked with $0.125 \mathrm{mg} / \mathrm{ml}$ pure LPS. (D) Overlay of all three spectra to identify similar peaks (LPS, green; oligo, red; spiked oligo, blue). Asterisks indicate distinct peaks seen in both LPS and LPS-spiked oligonucleotide but not in the non-spiked oligonucleotide. This identical overlap identifies peaks representing LPS in DNA samples

Figure 2. In vivo expression assay in mice lacking LPS recognition components. Mice are intra-articularly injected with $30 \mu g$ pCF1-Luc expression plasmid. Twenty-four hours post-injection luciferase levels are detected and quantified as photons/second with the Xenogen In vivo imaging system (IVIS). Expression levels are compared between wild-type control mice and mice lacking proteins of the LPS recognition complex; t-tests comparing these expression levels are used to determine $p$-values. For all images the top mouse is the wild-type control and the bottom mouse is the experimental. (A) LBP knockout and (B) CD14 knockout mice show a higher luciferase expression than from the wild-type controls. (C) TLR-4 knockout mice show no change in expression of luciferase level from wild-type mice. (D) In mice lacking CD44, a major receptor in synovial tissue, but which does not bind LPS, there is no difference in expression as compared to wild-type mice 

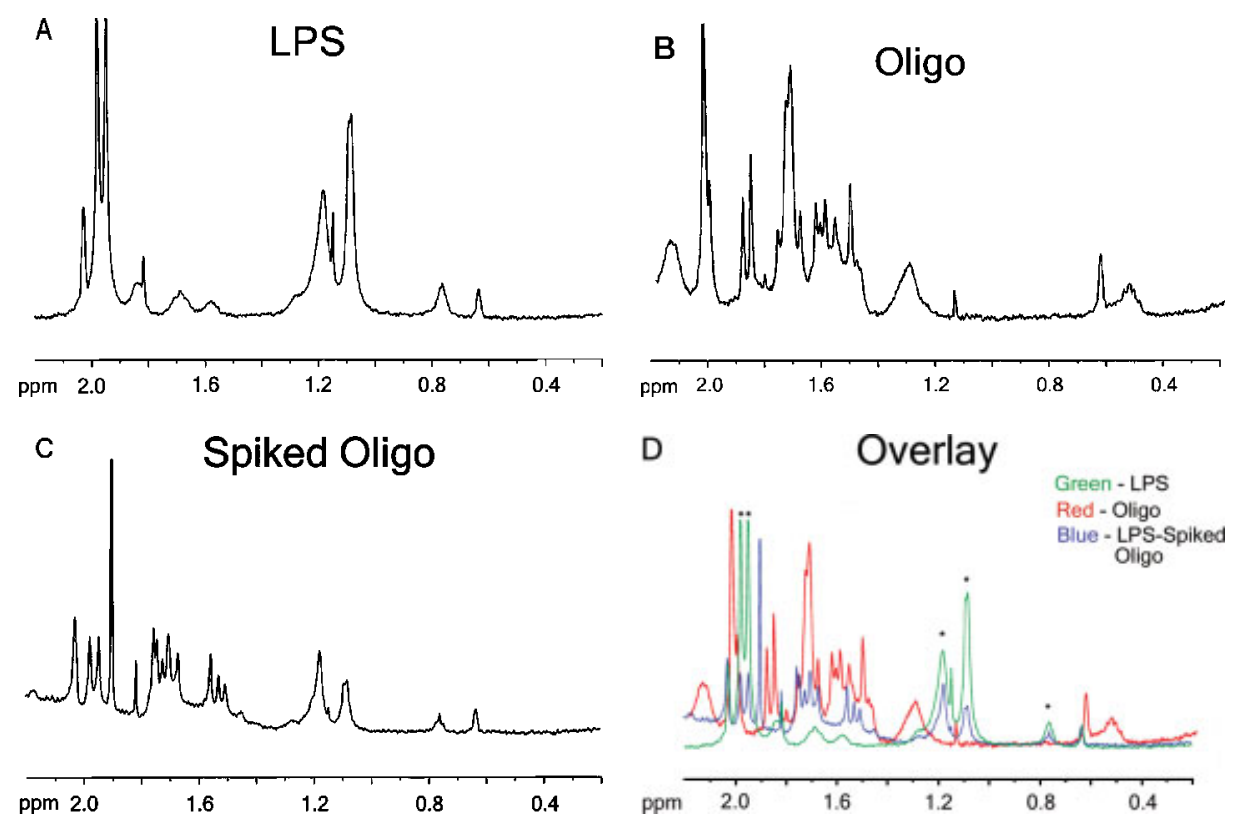

Figure 1.
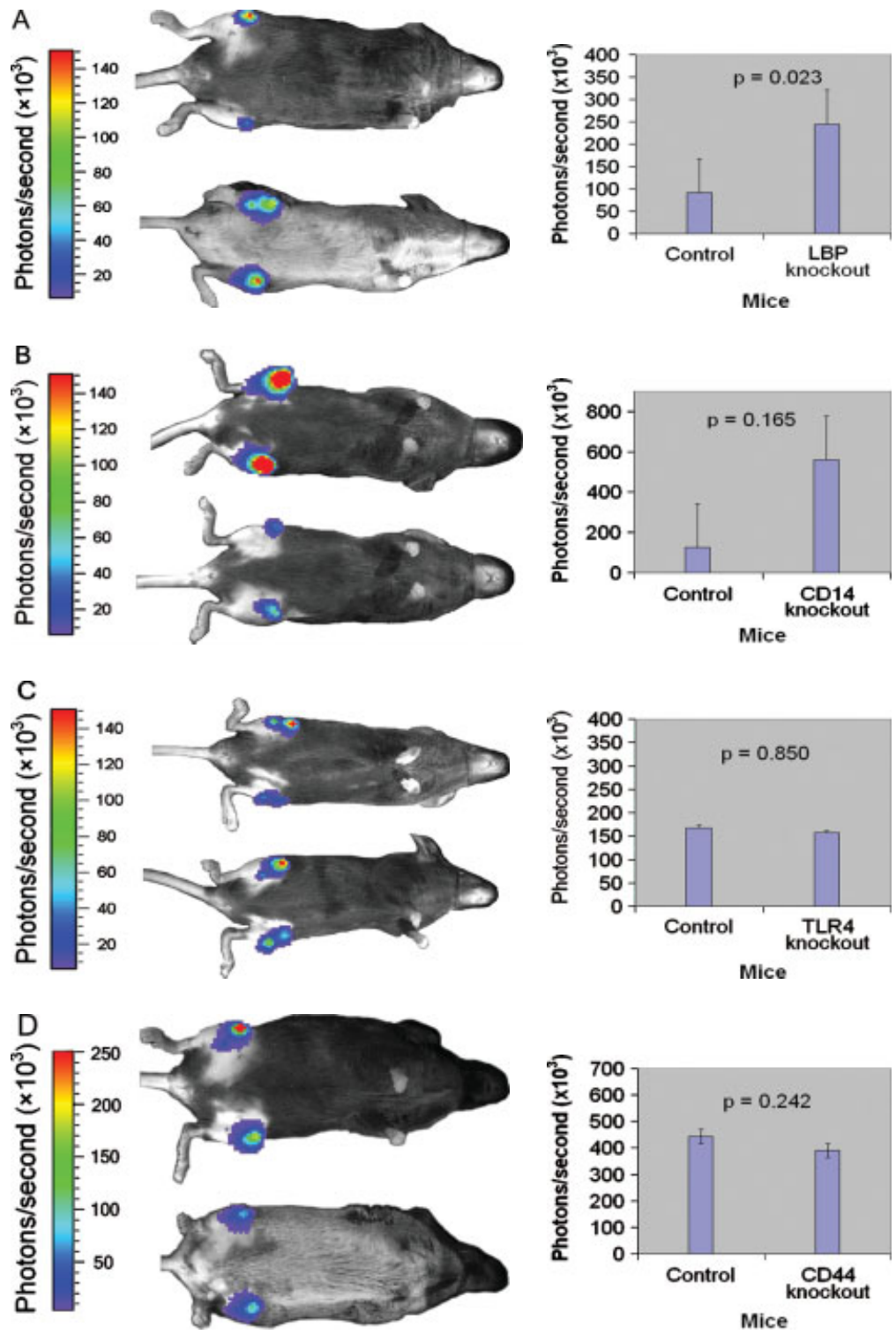

Figure 2. 

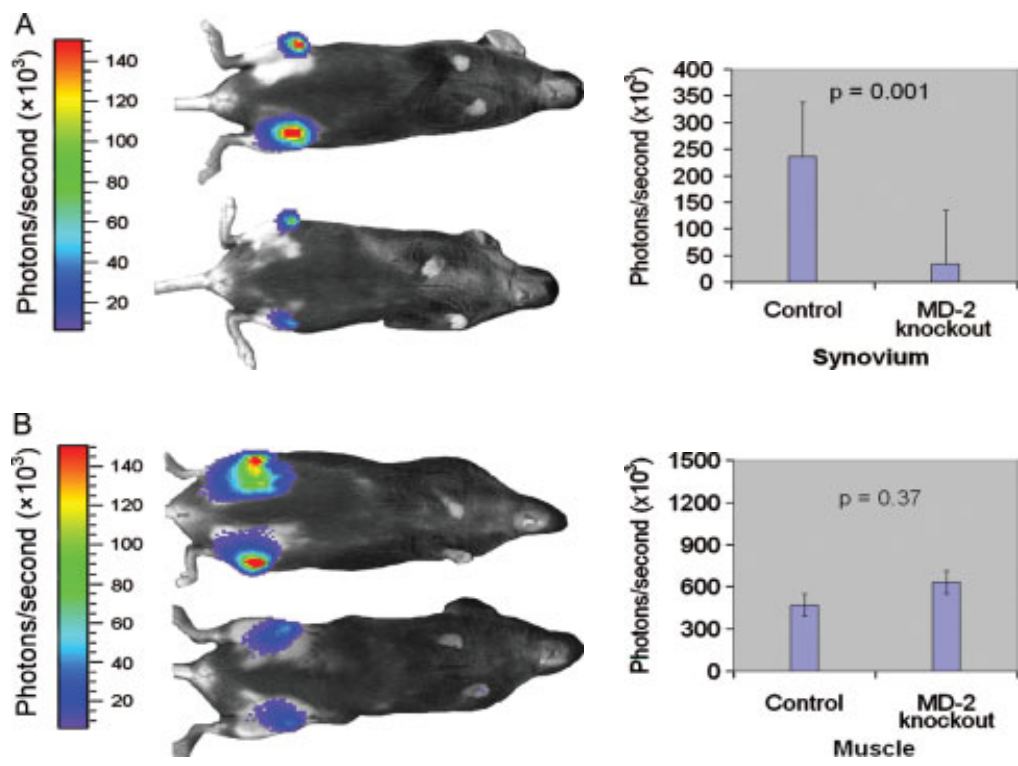

Figure 3. MD-2-mediated plasmid DNA uptake in synovium. Both wild-type and MD-2 -/- mice were injected with $30 \mu \mathrm{g}$ aqueous pCF1-Luc expression plasmid into (A) synovial fluid or (B) muscle tissue. In both images, the top mouse is the control and the bottom mouse is the experimental. In mice lacking MD-2 luciferase expression is significantly decreased in synovial tissue. In muscle tissue, which does not express MD-2, there is no significant difference in expression. Luciferase expression is detected $24 \mathrm{~h}$ following plasmid injection using the IVIS and quantified as photons/second; t-tests are used to determine $p$-values
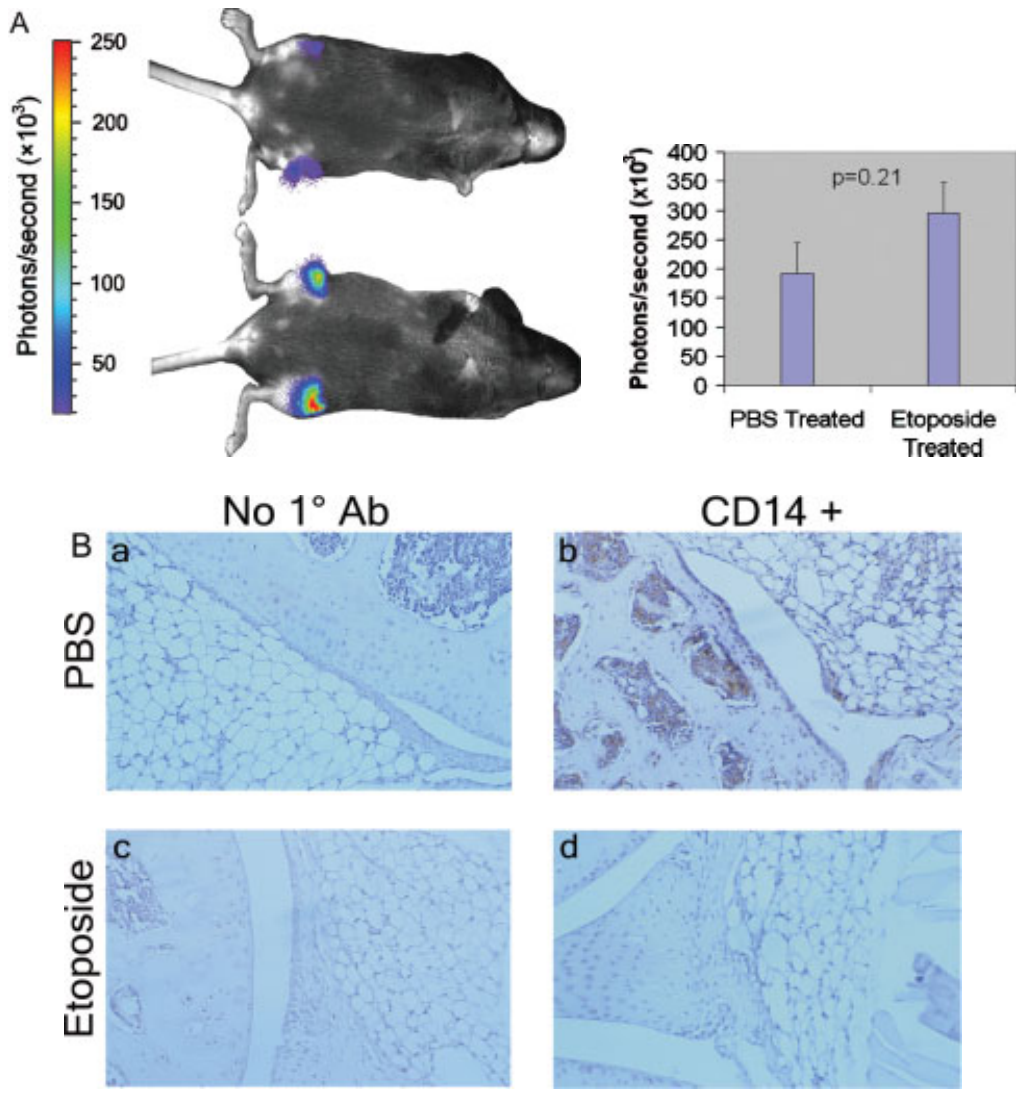

Figure 4. Fibroblast-like synoviocytes are the major cell type responsible for plasmid uptake and expression in synovial tissue. (A) C57BL/6J mice $(n=8)$ were treated by subcutaneous injection with either PBS (top mouse) or etoposide (bottom mouse) at $12.5 \mathrm{mg} / \mathrm{kg}$ for 3 consecutive days followed by intra-articular injection of plasmid DNA and bioluminescent imaging exactly as described above. A fourth treatment dose was additionally given on the day of imaging. Mice depleted of macrophages from the etoposide treatments exhibit increased luciferase expression compared to mice treated with PBS. Luciferase expression is quantified as photons/second by the IVIS, and mean values are compared exactly as described above. (B) Paraffin sections of stifle joints from etoposide- and PBS-treated mice were stained with purified anti-mouse CD14 antibody as a marker for macrophages. The synovial tissue from mice treated with PBS as control was positive for CD14 (b), but CD14 is not present in synovial tissue from mice treated with etoposide (d). (a, c) Synovial tissue from PBS- and etoposide-treated mice incubated without the CD14 antibody 
fluid. There were no statistically significant differences in luciferase expression between CD44 knockouts and wild-type controls. However, there was a trend toward increased levels of luciferase expression in CD44 knockout mice. This suggests the possibility that hyaluronic acid binding proteins may also modulate transfection of synovial cells via non-LPS-dependent mechanisms.

$\mathrm{MD}-2$, in addition to being an essential cofactor to TLR-4 for LPS signaling, has also been shown to bind LPS [38] and exist in a soluble form [39]. As a protein that is part of the LPS recognition complex and one that specifically binds LPS we examined the role that MD2 plays in plasmid uptake and expression by looking at luciferase expression levels of mice lacking MD-2 (Figure 3). Figure 3A compares luciferase expression of MD-2 knockout mice to wild-type controls after intraarticular injection, and shows there is a significant decrease in luciferase expression in mice lacking MD2 , suggesting a critical role for MD-2 in cytoplasmic uptake and expression of plasmid DNA. To identify possible tissue- and/or cell-specific utilization of MD-2 in plasmid DNA transfection we examined in vivo luciferase expression in skeletal muscle. Skeletal muscle has been extensively studied as a target for DNA transfection using naked plasmid DNA, and a variety of non-receptormediated endocytic mechanisms for cytoplasmic entry of plasmid DNA identified $[1,4,12,13,40]$. In addition, skeletal muscle has been shown not to express MD-2 [24]. Figure 3B shows the results of the luciferase assay in MD-2 knockout mice and wild-type control following intra-muscular injection of the pCF1-Luc plasmid. We observe no differences in luciferase expression in skeletal muscle between wild-type mice and mice lacking MD-2, suggesting that alternative pathways for plasmid DNA internalization predominate in this tissue. This also suggests that MD-2-mediated DNA uptake may be tissue and/or cell type specific.

In order to clarify the identity of transfected cells within the stifle joints we treated wild-type mice with etoposide to deplete macrophages prior to treatment with intraarticular pCF1-Luc plasmid followed by analysis with the bioluminescent in vivo expression assay. Figure 4A shows that mice depleted of macrophages show an increase, although not statistically significant, in luciferase expression as compared to mice treated with PBS as control. To confirm etoposide-mediated depletion of macrophages we harvested the stifle joints from treated and control mice. Paraffin sections (saggatil) were stained with anti-CD14 antibody as a marker for macrophages. Figure $4 \mathrm{~B}$ shows representative sections from the stifle joints of both PBS- and etoposide-treated mice. Panel (b) shows articular tissues from PBS-treated mice with extensive positive staining for CD14, indicating the presence of macrophages in both bone marrow and synovium. Mice treated with etoposide had no evidence of CD14-positive cells present within either the bone marrow or synovium (panel d) indicating successful depletion of macrophages. Control sections reacted with secondary antibody alone showed no evidence of background staining (panels a and c).

The intima of normal synovium is composed primarily of synovial fibroblasts and synovial macrophages, with very small percentages of resident dendritic cells and lymphocytes [41]. These results suggest that the majority of cellular uptake and expression of plasmid DNA following intra-articular administration occurs in fibroblast-like synoviocytes. However, it is also possible that some percentage of subintimal fibroblasts or endothelial cells of the subintimal vascular plexus may also be susceptible to in vivo transfection and may contribute to the overall level of luciferase expression observed in vivo [41].

\section{Discussion}

It has been shown in numerous studies that plasmid DNA can gain access to cytoplasm and nuclei of a variety of cell types in vivo [1,8-13]. In specific cells this occurs presumably through a process of receptormediated endocytosis. However, the identities of the cellular receptor(s) and mechanism(s) involved remain incompletely understood. In these studies we have begun to elucidate the role that $E$. coli-derived LPS, and the TLR4 accessory protein MD-2, play in the process of in vivo transfection.

Plasmid DNA isolated from $E$. coli has physically associated to it residual LPS from membranes of the Gram-negative bacteria. Despite extensive purification methods detectable amounts of functional LPS remain in aqueous solutions of highly purified plasmid DNA. Our data supports the hypothesis that there is a noncovalent complex formed between plasmid DNA and LPS. We believe that following in vivo administration the DNA-LPS complex is recognized and bound to the soluble LPS-binding proteins LBP, CD14, and MD-2. Our data show that LBP and CD14 function to downmodulate transfection whereas MD-2 appears to facilitate transfection.

It is clear from the results of the sensitive bioassay that small amounts of residual LPS are present in highly purified preparations of plasmid DNA. Our expectation was that the application of NMR spectroscopy and FAME analyses would allow us to more precisely quantify the amount of residual LPS present in association with highly purified plasmid DNA and determine the stoichiometric relationship between these components. Unfortunately, the levels of residual LPS present in highly purified preparations of plasmid DNA were either at or slightly below the levels of detection of both NMR and FAME analyses. In addition, these assays do not allow us to verify our hypothesis that LPS and plasmid DNA may exist in solution as a non-covalent complex, and that this complex may have distinct biological properties that are dependent on soluble proteins that are known to bind to LPS in biological fluids. Our results are not completely unexpected given the difficulty that other investigators 
have encountered in the application of biochemical or biophysical methods to characterize the structure-function relationships of LPS complexes [42]. At best we can use the limits of detection for the FAME analyses to provide us with an estimate of the levels of residual LPS. Based on the limits of detection of the FAME analyses, the absolute level of LPS per plasmid DNA is on the order of 1.6 molecules of LPS per molecule of $5.5 \mathrm{~kb}$ plasmid DNA. These analytical results provide indirect evidence for the existence of a DNA-LPS complex, which supports our in vivo data suggesting that plasmid DNA transfection is modulated by interactions with soluble LPS-binding proteins.

The in vivo bioluminescent expression experiments also provide strong, although indirect, evidence supporting the existence of complex formation between LPS and plasmid DNA. This physical association is made evident by the change in luciferase expression when plasmid DNA is administered in vivo in the absence of LPSbinding proteins. The normal functions of LBP and CD14 are to facilitate transfer of LPS to the TLR-4MD-2 complex to signal an innate immune response. The increase in luciferase expression observed in mice deficient in either LBP or CD14 and the decrease in expression in mice lacking MD-2 suggest that LBP and CD14 compete with MD-2 for binding to plasmid DNA through its association with LPS. Disparity between the mechanisms of LPS signaling and endocytosis has been previously recognized in several studies $[35,43,44]$. Our results are consistent with previous studies showing that $\mathrm{CD} 14$, in particular, functions as a competitive inhibitor for endocytosis of purified LPS, and support the hypothesis that soluble MD-2 is the protein that is responsible for endocytosis of LPS. The magnitude of competition between soluble CD14 and soluble MD-2 is not known; however, our in vivo results are consistent with the reported LPS-binding affinities for $\mathrm{LBP}\left(\mathrm{K}_{\mathrm{D}}=3.5 \times 10^{-9}\right)$ and $\operatorname{sCD} 14\left(\mathrm{~K}_{\mathrm{D}}=30-74 \times 10^{-9}\right)[21,45]$. The binding affinity between LPS and LBP is approximately 10 to 20 times greater than that between LPS and CD14, which is reflected in the statistically, and non-statistically, significant increase in luciferase expression in LBP- and CD14-deficient mice, respectively. In the absence of either LBP or CD14 we see a significant bias towards LPS internalization and a resultant increase in luciferase expression. This increase in protein expression observed in the LBP and CD14 knockout mice supports the hypothesis of complex formation between LPS and E. coli-derived plasmid DNA.

MD-2 has proven to be required for proper cellular distribution, and LPS recognition, by TLR-4. MD-2 has also been shown to exist in a soluble form that can bind LPS with a high binding affinity $\left(\mathrm{K}_{\mathrm{D}}=65 \times 10^{-9}\right)$, similar to that exhibited by CD14 [38,45]. Mice lacking MD-2 exhibited a significant decrease in luciferase expression in vivo following intra-articular, but not intra-muscular, administration of pCF1-Luc. This decrease in luciferase expression observed in synovial tissue of MD-2 knockout mice suggests that MD-2 in synovial tissue, probably soluble MD-2 within synovial fluid, has an important involvement in mediating internalization of plasmid DNA through its ability to bind LPS. It is important to note that skeletal muscle cells do not express high levels of LPS-binding proteins including MD-2. In skeletal muscle of MD-2 knockout mice we observed no change in plasmid uptake and luciferase expression in the absence of MD-2. This result supports the hypothesis that several nonendocytic pathways of plasmid DNA uptake predominate in skeletal muscle, and it supports the probable role of soluble MD-2 within the synovial fluid. A direct pathway by which synovial MD-2 binds to and mediates internalization of LPS-DNA complexes is consistent with the increases in luciferase expression that were observed in the LBP and CD14 knockout mice. In this model LBP and CD14 function as competitive inhibitors of MD-2.

In summary, we have identified a potential in vivo mechanism that is used by E. coli-derived plasmid DNA to gain entry into fibroblast-like synoviocytes that is dependent on the LPS-binding protein MD-2. Further, our data indicate that LBP and soluble CD14 function as competitive inhibitors of in vivo transfection. These results have important implications for the use of plasmid DNA as both a clinical gene transfer vector system and a vaccine. Many studies have examined the use of $E$. coli-derived plasmid DNA as a vaccine; however, in vivo results have often been inconsistent. It is interesting to speculate that local concentrations of LBP, sCD14, and MD-2 may influence pharmacodistribution of plasmid DNA between signaling and endocytic pathways for LPS processing used by antigen-presenting cells. In turn these tissue- or cell-specific effects may influence global host immune responses at both the innate and adaptive level.

\section{Acknowledgements}

This study was supported by grants from the US National Institutes of Health, R01 HL053713, R24 CA83099, and P30 AR48310. We thank Dan Hall and Brian Ross of the Michigan Small Animal Imaging Resource for assistance in the bioluminescent imaging, the staff of the University of Michigan Vector Core for production of highly purified pCF1-Luc, and Cynthia Marcelo for assistance with the analysis of FAMEs.

\section{References}

1. Wolff J, Malone R, Williams P, et al. Direct gene transfer into mouse muscle in vivo. Science 1990; 247: 1465-1468.

2. Danko I, Williams $\mathrm{P}$, Herweijer $\mathrm{H}$, et al. High expression of naked plasmid DNA in muscles of young rodents. Hum Mol Genet 1997; 6: 1435-1443.

3. Davis HL, Demeneix BA, Quantin B, et al. Plasmid DNA is superior to viral vectors for direct gene transfer into adult mouse skeletal muscle. Hum Gene Ther 1993; 4: 733-740.

4. Wolff JA, Ludtke JJ, Acsadi G, et al. Long-term persistence of plasmid DNA and foreign gene expression in mouse muscle. Hum Mol Genet 1992; 1: 363-369.

5. Liu F, Liang KW, Huang L. Systemic administration of naked DNA: gene transfer to skeletal muscle. Mol Interv 2001; 1: 168-172. 
6. Sikes ML, O'Malley BW Jr, Finegold MJ, et al. In vivo gene transfer into rabbit thyroid follicular cells by direct DNA injection. Hum Gene Ther 1994; 5: 837-844.

7. McArthur CP, Wang Y, Heruth D, et al. Amplification of extracellular matrix and oncogenes in tat-transfected human salivary gland cell lines with expression of laminin, fibronectin, collagens i, iii, iv, c-myc and p53. Arch Oral Biol 2001; 46: 545-555.

8. $\mathrm{Wu} \mathrm{H,} \mathrm{Ramachandran} \mathrm{C,} \mathrm{Bielinska} \mathrm{AU,} \mathrm{et} \mathrm{al.} \mathrm{Topical}$ transfection using plasmid DNA in a water-in-oil nanoemulsion. Int J Pharm 2001; 221: 23-34.

9. Choate KA, Khavari PA. Direct cutaneous gene delivery in a human genetic skin disease. Hum Gene Ther 1997; 8: 1659-1665.

10. Budker V, Zhang G, Knechtle S, et al. Naked DNA delivered intraportally expresses efficiently in hepatocytes. Gene Therapy 1996; 3: 593-598.

11. Zhang G, Vargo D, Budker V, et al. Expression of naked plasmid DNA injected into the afferent and efferent vessels of rodent and dog livers. Hum Gene Ther 1997; 8: 1763-1772.

12. Nita I, Ghivizzani SC, Galea-Lauri J, et al. Direct gene delivery to synovium. An evaluation of potential vectors in vitro and in vivo. Arthritis Rheum 1996; 39: 820-828.

13. Ohashi S, Kubo T, Kishida T, et al. Successful genetic transduction in vivo into synovium by means of electroporation. Biochem Biophys Res Commun 2002; 293: 1530-1535.

14. Budker V, Budker T, Zhang G, et al. Hypothesis: naked plasmid DNA is taken up by cells in vivo by a receptor-mediated process. J Gene Med 2000; 2: 76-88.

15. Quezada A, Larson J, French M, et al. Biodistribution and safety studies of hdel-1 plasmid-based gene therapy in mouse and rabbit models. J Pharm Pharmacol 2004; 56: 177-185.

16. Seydel U, Oikawa M, Fukase K, et al. Intrinsic conformation of lipid A is responsible for agonistic and antagonistic activity. Eur J Biochem 2000; 267: 3032-3039.

17. Holst O, Muller-Loennies S, Lindner B, et al. Chemical structure of the lipid A of Escherichia coli j-5. Eur J Biochem 1993; 214: 695-701.

18. Center for Biologics Evaluation and Research. Guideline on validation of the limulus amebocyte lysate test as an endproduct endotoxin test for human and animal parenteral drugs, biological products, and medical devices. United States Food and Drug Administration: Rockville, MD, 1987.

19. Schumann R, Leong S, Flaggs G, et al. Structure and function of lipopolysaccharide binding protein. Science 1990; 249: 1429-1431.

20. Tapping RI, Tobias PS. Cellular binding of soluble cd14 requires lipopolysaccharide (LPS) and LPS-binding protein. $J$ Biol Chem 1997; 272: 23 157-23 164.

21. Tobias P, Soldau K, Gegner J, et al. Lipopolysaccharide binding protein-mediated complexation of lipopolysaccharide with soluble cd14. J Biol Chem 1995; 270: 10 482-10 488.

22. Wright SD, Ramos RA, Tobias PS, et al. Cd14, a receptor for complexes of lipopolysaccharide (LPS) and LPS binding protein. Science 1990; 249: 1431-1433.

23. da Silva Correia J, Soldau K, Christen U, et al. Lipopolysaccharide is in close proximity to each of the proteins in its membrane receptor complex. Transfer from CD14 to TLR4 and MD-2. $J$ Biol Chem 2001; 276: 21 129-21 135

24. Shimadzu R, Akashi S, Ogata H, et al. MD-2, a molecule that confers lipopolysaccharide responsiveness on toll-like receptor 4. J Exp Med 1999; 189: 1777-1782.

25. Nagai Y, Akashi S, Nagafuku M, et al. Essential role of MD-2 in LPS responsiveness and TLR4 distribution. Nat Immunol 2002; 3: 667-672.

26. Mancek M, Pristovsek P, Jerala R. Identification of LPS-binding peptide fragment of MD-2, a toll-receptor accessory protein. Biochem Biophys Res Commun 2002; 292: 880-885.
27. Yovandich J, O'Malley Jr. B, Sikes M, et al. Gene transfer to synovial cells by intra-articular administration of plasmid DNA. Hum Gene Ther 1995; 6: 603-610.

28. Raczka E, Kukowska-Latallo JF, Rymaszewski M, et al. The effect of synthetic surfactant exosurf on gene transfer in mouse lung in vivo. Gene Ther 1998; 5: 1333-1339.

29. von Knethen A, Lotero A, Brune B. Etoposide and cisplatin induced apoptosis in activated raw 264.7 macrophages is attenuated by camp-induced gene expression. Oncogene 1998; 17: $387-394$

30. Barland P, Novikoff AB, Hamerman D. Electron microscopy of the human synovial membrane. J Cell Biol 1962; 14: 207-220.

31. Ghadially FN. Fine Structure of Synovial Joints: A Text and Atlas of the Ultrastructure of Normal and Pathological Articular Tissues, Butterworths: Boston, 1983.

32. Gilbert JC, Martin SF. Experimental Organic Chemistry: A Miniscale and Microscale Approach, Saunders College Publishing: Orlando, FL, 1998.

33. Loppnow H, Brade H, Durrbaum I, et al. Il-1 induction-capacity of defined lipopolysaccharide partial structures. J Immunol 1989; 142: 3229-3238.

34. Rietschel ET, Kirikae T, Schade FU, et al. Bacterial endotoxin: molecular relationships of structure to activity and function. FASEB J 1994; 8: 217-225.

35. Kitchens RL, Munford RS. CD14-dependent internalization of bacterial lipopolysaccharide (LPS) is strongly influenced by LPS aggregation but not by cellular responses to LPS. J Immunol 1998; 160: 1920-1928.

36. Dunzendorfer S, Lee HK, Soldau K, et al. TLR4 is the signaling but not the lipopolysaccharide uptake receptor. J Immunol 2004; 173: 1166-1170.

37. Hale LP, Haynes BF, McCachren SS. Expression of CD44 variants in human inflammatory synovitis. J Clin Immunol 1995; 15: 300-311.

38. Viriyakosol S, Tobias PS, Kitchens RL, et al. MD-2 binds to bacterial lipopolysaccharide. J Biol Chem 2001; 276: 38 044-38 051 .

39. Visintin A, Mazzoni A, Spitzer JA, et al. Secreted MD-2 is a large polymeric protein that efficiently confers lipopolysaccharide sensitivity to toll-like receptor 4. Proc Natl Acad Sci U S A 2001; 98: 12 156-12 161 .

40. Davis HL, Whalen RG, Demeneix BA. Direct gene transfer into skeletal muscle in vivo: factors affecting efficiency of transfer and stability of expression. Hum Gene Ther 1993; 4: 151-159.

41. Edwards JCW. Functions of synovial lining. In The Synovial Lining in Health and Disease, Henderson B, Edwards JCW (eds). Chapman \& Hall: London, 1987; 41-74.

42. Wang Y, Hollingsworth RI. An NMR spectroscopy and molecular mechanics study of the molecular basis for the supramolecular structure of lipopolysaccharides. Biochemistry 1996; 35: 5647-5654.

43. Latz E, Visintin A, Lien E, et al. Lipopolysaccharide rapidly traffics to and from the Golgi apparatus with the toll-like receptor 4-MD-2-CD14 complex in a process that is distinct from the initiation of signal transduction. $J$ Biol Chem 2002; 277: $47834-47843$.

44. Suzuki M, Hisamatsu T, Podolsky DK. Gamma interferon augments the intracellular pathway for lipopolysaccharide (LPS) recognition in human intestinal epithelial cells through coordinated up-regulation of LPS uptake and expression of the intracellular toll-like receptor 4-MD-2 complex. Infect Immunol 2003; 71: 3503-3511.

45. Viriyakosol S, Mathison JC, Tobias PS, et al. Structure-function analysis of CD14 as a soluble receptor for lipopolysaccharide. $J$ Biol Chem 2000; 275: 3144-3149. 